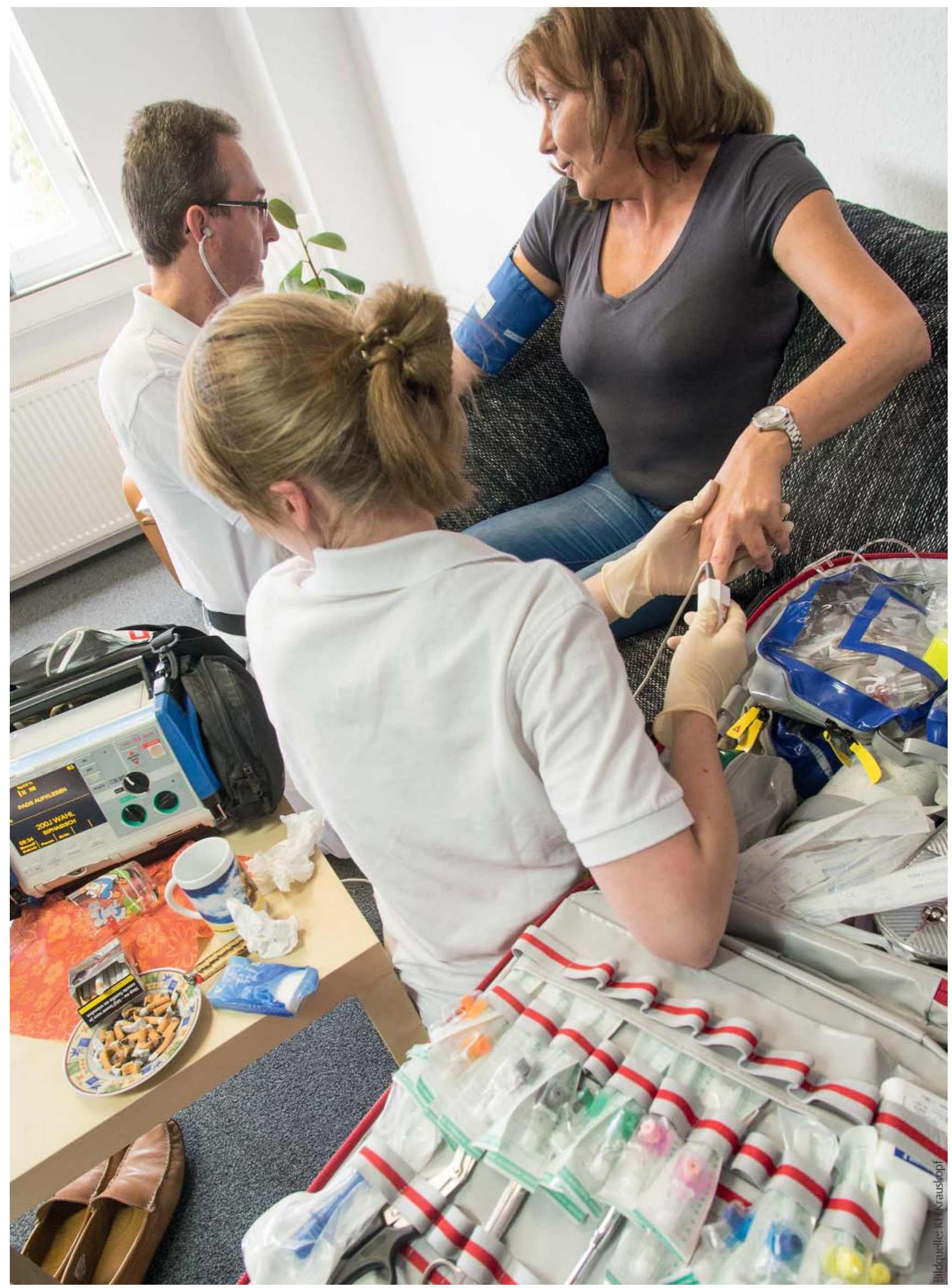




\section{Exazerbierte COPD - mehr als nur Atemnot}

Jochen Hinrichs-Pavlik, Jule Pavlik

\section{Im Rettungsdienst wird man häufig zu Patienten gerufen, die keine Luft mehr} bekommen. Kritisch wird es aber, wenn sich deren Zustand weiter verschlechtert, es zu einem ventilatorischen Versagen kommt oder die Patienten bewusstlos werden. Oft liegen auch noch zusätzliche Begleiterkrankungen vor, die die Situation weiter verschärfen. Wie Sie eine akute Verschlechterung einer chronisch-obstruktiven Lungenerkrankung (COPD) von anderen möglichen Krankheiten unterscheiden können und wie Sie schnell und richtig handeln, lesen Sie in diesem Beitrag.

\section{Akute Atemnot}

\section{FALLBEISPIEL}

Der Rettungsdienst wird von Frau S. zu ihrem Ehemann alarmiert, da er zunehmende Atemnot hat. Er ist 61 Jahre alt, und bei ihm ist seit Jahren eine mittelschwere COPD II. Grades bekannt. Seit 4 Tagen leide er unter einer zunehmenden Kurzatmigkeit inzwischen schon in Ruhe - sowie unter trockenem Reizhusten. Momentan hat der Patient weder Fieber noch Schmerzen im Brustkorb. Von seiner Frau wurde eine zunehmende Vergesslichkeit beobachtet. Beim Eintreffen des Rettungsteams wirkt Herr S. verlangsamt und leicht verwirrt. Die Atemfrequenz beträgt 24/min, eine leichte Lippenzyanose ist erkennbar, die pulsoxymetrisch gemessene Sauerstoffsättigung bei Raumluft $\left(\mathrm{SpO}_{2}\right.$ ) liegt bei $80 \%$ (Norm $>94 \%$ ), der Puls ist mit 106/min rhythmisch, der Blutdruck beträgt $130 / 80 \mathrm{mmHg}$. Es sind leichte Unterschenkelödeme erkennbar. Beim Abhören stellen Sie ein schwaches Atemgeräusch mit Giemen und Brummen beidseits fest.

Das Team verabreicht sofort $3 \mathrm{l} / \mathrm{min}$ Sauerstoff über eine Nasenbrille, worauf die Sauerstoffsättigung auf $90 \%$ ansteigt. Der Patient erhält einen venösen Zugang und vom inzwischen eingetroffenen Notarzt Prednisolon $100 \mathrm{mg}$ i. v. Herr S. bekommt zusätzlich 2 Hübe Salbutamol über einen Spacer (Inhalationshilfe). Die Rettungskräfte bringen Herrn S. im RTW mit Notarztbegleitung in eine Lungenfachklinik.

Bei der stationären Aufnahme auf die pneumologische Wachstation bestätigt sich die vermutete infektexazerbierte COPD mit ventilatorischem Versagen. Eine Blutgasanalyse unter Sauerstoffgabe zeigt einen leicht erniedrigten Sauerstoffpartialdruck $\left(\mathrm{pO}_{2}\right)$ mit $64 \mathrm{mmHg}($ Norm $>72 \mathrm{mmHg})$ und einen deutlich erhöhten Kohlendioxidpartialdruck $\left(\mathrm{pCO}_{2}\right)$ mit $67 \mathrm{mmHg}$ (Norm 35-45 mmHg). Die Kortisontherapie wird oral fortgesetzt und bei erhöhten Infektparametern im Labor durch eine Antibiose ergänzt. Außerdem erhält Herr S. zusätzlich Diuretika. Die inhalative Therapie und die Atemtherapie werden intensiviert und eine nichtinvasive Beatmung (NIV) wird eingeleitet. Herr S. toleriert diese gut. Seine Blutgase bessern sich langsam, v. a. der $\mathrm{pCO}_{2}$. Nach 14 Tagen kann Herr S. in stabilem Allgemeinzustand nach Hause gehen. Er ist im Alltag altersentsprechend belastbar und setzt nachts die NIV selbstständig und ohne Probleme fort.

\section{Ursachen akuter Atemnot}

Plötzliche Atemnot ist eines der häufigsten Symptome in der Akutmedizin. Ohne Sauerstoff kann der Organismus nur wenige Minuten ohne irreversible Schäden überleben. Folgende Ursachen können u. a. eine akute Atemnot auslösen:

- Verlegung der Atemwege: z. B. durch Bolusaspiration, Larynxödem z. B. nach Insektenstich

- pulmonale Ursachen: z. B. aufgrund akuter bronchialer Obstruktion bei Asthmaanfall oder bei exazerbierter COPD, Pneumothorax

- blockierter Sauerstofftransport: z. B. durch Vergiftungen (Kohlenmonoxidvergiftung)

\section{Differenzialdiagnosen}

Kardiale Ursachen

Differenzialdiagnostisch muss man auch kardiovaskuläre Ursachen beachten:

- Myokardinfarkt

- Rechts-, Linksherzinsuffizienz

- Lungenembolie 
- Tab. 1 Unterscheidung von Patienten mit Asthma und COPD.

\begin{tabular}{|l|l|l|}
\hline & Asthma & COPD \\
\hline Alter & $\begin{array}{l}\text { überwiegend jung } \\
(<50 \jmath .)\end{array}$ & $\begin{array}{l}\text { überwiegend älter } \\
(>50] .)\end{array}$ \\
\hline Tabakkonsum & selten & sehr häufig \\
\hline Auswurf & wenig, glasig & zäh, z. T. eitrig \\
\hline Allergien & häufig (Trigger) & selten \\
\hline Infekte & möglich & häufig (Trigger) \\
\hline Besserung der & meist vollständig & nur wenig \\
\hline Obstruktion & nur im Anfall & mit dem Alter \\
\hline Luftnot & zunehmend \\
\hline Beginn der Luftnot & Minuten bis Stunden & Stunden bis Tage \\
\hline Auslöser & Allergien, Infekte & Infekte \\
\hline
\end{tabular}

\section{Asthma bronchiale}

Immer häufiger trifft der Rettungsdienst auf Patienten mit einer obstruktiven Atemwegserkrankung. Darunter versteht man im Wesentlichen die chronisch-obstruktive Bronchitis und das Lungenemphysem. Diese werden unter dem Begriff COPD zusammengefasst. Davon abzugrenzen ist das Asthma bronchiale. Dem Asthma liegt eine andere Krankheitsursache zugrunde. Es ist gekennzeichnet durch eine hyperreagible Bronchialschleimhaut. Sie reagiert auf äußere Reize (Allergene, starke körperliche Aktivität, Stress etc.) mit einer Hypersekretion und einer variablen Enge der Bronchien (Spasmus). Auch die medikamentöse Therapie hat andere Ansatzpunkte - sie führt im Gegensatz zur COPD-Therapie häufig zu einer raschen Besserung der Beschwerden.

\section{Merke}

Asthma und COPD sind 2 grundsätzlich unterschiedliche Erkrankungen mit ganz unterschiedlicher Therapie.

\section{COPD}

\section{Überblick}

\section{Eine Volkskrankheit}

Die COPD gewinnt in den letzten Jahren zunehmend an Bedeutung und gilt inzwischen als eine der häufigsten Erkrankungen. Schätzungen zufolge leiden 10 - 15\% der Erwachsenen in Deutschland an einer COPD. Weltweit ist die COPD die vierthäufigste Todesursache. Sie ist charakterisiert durch eine chronisch fortschreitende Verengung der Bronchien, die sich auch unter Therapie nicht normalisiert.

\section{COPD vs. Asthma}

Durch die chronische Enge der Bronchien, die sog. Obstruktion, unterscheidet sie sich grundsätzlich vom Asthma bronchiale ( $\vee$ Tab. 1 ). Das Asthma bronchiale ist durch eine reversible Obstruktion der Atemwege charakterisiert, und mittels entsprechender Medikamente lassen sich normale Lungenfunktionswerte erreichen.

\section{Häufigste Ursache: Rauchen}

\section{Merke}

Es gibt viele Auslöser für eine COPD, der wichtigste ist das Zigarettenrauchen!

Durch langjährigen Nikotinkonsum kommt es zu einer chronischen Reizung der Bronchialschleimhaut. Diese reagiert mit einer chronischen Entzündung, geprägt durch Schwellung, Schleimproduktion und eine Kontraktion der Bronchialmuskulatur. Durch diese Faktoren verengen sich die Bronchien und behindern den Atemstrom. Zusätzlich führen die chronischen Entzündungsreaktionen über einen längeren Zeitraum zu einem Elastizitätsverlust der Bronchien. Es tritt eine kaum noch reversible Obstruktion auf (Remodeling). Bei starker Ausatmung kann es zusätzlich zu einem Kollaps der Bronchien kommen.

\section{Symptome}

Patienten mit einer COPD leiden typischerweise unter Kurzatmigkeit (Dyspnoe), anfangs nur bei Anstrengung, in fortgeschrittenen Fällen auch in Ruhe. Außerdem können Symptome einer chronischen Bronchitis (Husten und Auswurf) vorliegen.

\section{Begleiterkrankungen}

Häufig haben COPD-Patienten weitere chronische Erkrankungen, die die COPD zusätzlich negativ beeinflussen. Oft bestehen z. B. Bluthochdruck, koronare Herzerkrankungen, Herzinsuffizienz und Stoffwechselerkrankungen wie Diabetes mellitus und Übergewicht. Fast die Hälfte aller COPD-Patienten hat neben ihrer Atemwegserkrankung bis zu 4 weitere internistische Krankheiten [1].

\section{Merke \\ Bei COPD-Patienten finden sich häufig weitere Erkran- kungen, die bei einer Exazerbation den akuten Notfall negativ beeinflussen.}

\section{Schweregradeinteilung}

Die Schweregradeinteilung der COPD erfolgt in erster Linie anhand der Flusslimitierung in den Atemwegen. Diese wird mit einem Lungenfunktionstest (Spirometrie) ermittelt. Dabei misst man die Einsekundenkapaziät (FEV1) - also die Luftmenge, die ein Patient bei maxima- 


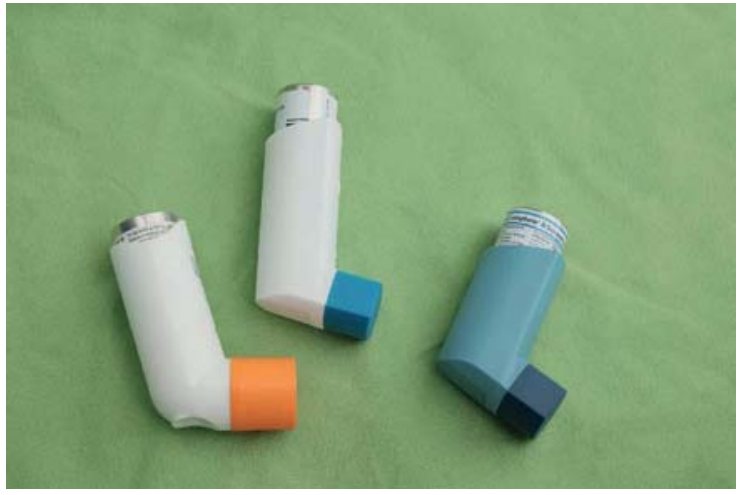

- Abb. 1 Auswahl an verschiedenen Dosieraerosolen (Bronchialöffner).

ler Ausatmung in $1 \mathrm{~s}$ ausatmen kann. Die Angabe erfolgt in Prozent, bezogen auf die individuellen Normwerte (alters-, geschlechts- und größenabhängig). Eine FEV1 von $50 \%$ bedeutet, dass die Bronchien nur noch zu $50 \%$ geöffnet sind. International wird der Schweregrad der COPD nach GOLD (Global Initiative for Chronic Obstructive Lung Disease) klassifiziert ( $\triangleright$ Tab. 2).

Im Alltag zeigt sich, dass nur wenige Patienten den Schweregrad ihrer COPD-Erkrankung kennen. Dies liegt z. T. auch daran, dass die Faktoren, die für die Schweregradeinteilung herangezogen werden, in den letzten Jahren mehrfach geändert wurden.

\section{PRAXIS/MAßNAHMEN}

\section{Fazit für die Praxis}

Die COPD ist eine langsam fortschreitende Erkrankung, an die sich die Patienten in einem gewissen Umfang anpassen können. So stimmt der gemessene Schweregrad nicht unbedingt mit den Symptomen oder den empfundenen Beeinträchtigungen im Alltag überein. Auch führt eine erniedrigte Sauerstoffsättigung nicht zwingend zu stärkeren Beschwerden.

\section{Therapie der stabilen COPD}

\section{Dosieraerosole}

Zur Therapie der COPD kommen v. a. inhalierbare Medikamente in Form von Sprays ( $\mathbf{A} \mathbf{b b} \mathbf{b} \mathbf{1}$ ) und Pulverinhalatoren zum Einsatz. An erster Stelle stehen dabei die sog. Bronchialöffner - auch Bronchodilatatoren oder Bronchospasmolytika genannt. Es gibt die Bedarfsmedikamente, die häufig von den Patienten als „Notfallspray“ bezeichnet werden, und die Basismedikamente.

Die Bedarfstherapie besteht meistens aus kurz wirksamen Bronchialöffnern ( $\beta_{2}$-Sympatomimetika), die man auch als SABA (short-acting beta agonist) bezeichnet.
- Tab. 2 Klassifikation des Schweregrads einer Obstruktion (FEV1).

\begin{tabular}{|l|l|l|}
\hline Schweregrad & Beeinträchtigung & $\begin{array}{l}\text { Einschränkung der } \\
\text { Bronchien }\end{array}$ \\
\hline GOLD 1 & leicht & $\geq 80 \%$ \\
\hline GOLD 2 & mäßig & $\geq 50$ bis $<80 \%$ \\
\hline GOLD 3 & schwer & $\geq 30$ bis $<50 \%$ \\
\hline GOLD 4 & sehr schwer & $<30 \%$ \\
\hline
\end{tabular}

D Tab. 3 Kurz wirksame Bronchialöffner.

\begin{tabular}{|l|l|}
\hline Wirkstoff & $\begin{array}{l}\text { Handelsname } \\
\text { (Beispiele) }\end{array}$ \\
\hline Salbutamol (SABA) & Salbutamol $^{\circledR} /$ Sultanol $^{\circledR}$ \\
\hline Fenoterol (SABA) & Berotec $^{\circledR}$ \\
\hline Ipratropiumbromid (SAMA) & Atrovent $^{\circledR}$ \\
\hline Fenoterol + Ipratropiumbromid & Berodual $^{\circledR}$ \\
\hline
\end{tabular}

Die SAMA (short-acting muscarinic antagonist) wiederum sind kurz wirksame Parasympatholytika, deren Wirkung erst nach ca. 15 - 20 min eintritt. Wichtigste Nebenwirkungen sind Herzfrequenzerhöhung, Unruhe und Tremor.

Die Basistherapie besteht aus lang wirksamen Bronchodilatatoren, die ebenfalls zur Gruppe der $\beta_{2}$-Sympatomimetika gehören (LABA, long-acting beta agonist), sowie aus Parasympatholytika (LAMA, long-acting muscarinic antagonist). Die Anticholinergika haben wesentlich geringere kardiale Nebenwirkungen, verursachen dafür Mundtrockenheit und führen in seltenen Fällen zu einem Harnverhalt. Häufig werden beiden Substanzklassen kombiniert ( $\triangleright$ Tab. 3).

Meist leiden COPD-Patienten auch an einem zähen, hochviskösen Schleim. Dann werden in der Basistherapie häufig elektrische Inhalationsgeräte zur Vernebelung von isotoner 0,9\%iger und hypertoner 3 \%iger Kochsalzlösung verordnet. Darüber können auch Salbutamol, Atrovent ${ }^{\circledR}$ oder Mischungen aus beiden Substanzen (Ipra$\mathrm{mol}^{\circledR}$ ) inhaliert werden.

Glukokortikoide (Kortison) kommen inhalativ nur bei der schweren COPD zum Einsatz sowie in Form von Tabletten und intravenös im Rahmen einer Exazerbation. Theophyllin hat seine Bedeutung in der Therapie der COPD verloren, Gründe dafür sind kardiale Nebenwirkungen und die ausgesprochen geringe therapeutische Breite. 
- Tab. 4 Abschätzen des COPD-Schweregrads.

\begin{tabular}{|c|c|c|c|c|}
\hline & Leicht & Mäßig & Schwer & $\begin{array}{l}\text { Sehr } \\
\text { schwer }\end{array}$ \\
\hline $\begin{array}{l}\text { inhalative } \\
\text { Bedarfstherapie }\end{array}$ & + & + & + & + \\
\hline LABA & & + & + & + \\
\hline LAMA & & + & + & + \\
\hline $\begin{array}{l}\text { inhalatives } \\
\text { Kortison }\end{array}$ & & & + & + \\
\hline Kortison Tbl. & & & & + \\
\hline $\begin{array}{l}\text { häuslicher } \\
\text { Sauerstoff }\end{array}$ & & & + & + \\
\hline Beatmung (NIV) & & & & + \\
\hline
\end{tabular}

\section{Merke}

Inhalative Bronchodilatatoren können die Pulsfrequenz und den Blutdruck erhöhen. Kontrollieren Sie deshalb regelmäßig die Vitalzeichen.

\section{Schweregrad erkennen}

\section{Respiratorisches Versagen}

Bei einer schweren COPD kommt es zu einem dauerhaft erniedrigten Sauerstoffpartialdruck im Blut. Diese Störung im Gasaustausch bezeichnet man als respiratorisches Versagen $\left(\mathrm{pO}_{2}\right.$ niedrig, $\mathrm{pCO}_{2}$ normal).

\section{Ventilatorisches Versagen}

Kommt es im Verlauf einer schweren COPD zu einer Überlastung der Atemmuskelpumpe, spricht man von einem ventilatorischen Versagen ( $\mathrm{pO}_{2}$ niedrig, $\mathrm{pCO}_{2}$ erhöht). Dabei ist die Atemmuskulatur erschöpft, was durch den Einsatz der Atemhilfsmuskulatur (Hals- und Brustmuskulatur) erkennbar ist. Die Angehörigen bemerken, dass der Patient unkonzentriert und zunehmend müde wirkt. Im Extremfall schläft er ein und ist nur noch schwer bis kaum erweckbar ( $\mathrm{CO}_{2}$-Narkose). Wie sich der aktuelle Schweregrad einer COPD abschätzen lässt, zeigt > Tab. 4.

\section{Merke}

Eine $\mathrm{CO}_{2}$-Narkose beschreibt eine Bewusstlosigkeit infolge einer zu hohen Konzentration von Kohlenstoffdioxid im Blut, beginnend bei $\mathrm{pCO}_{2}>60 \mathrm{mmHg}$.

\section{Exazerbation}

\section{Symptome}

Der Verlauf einer COPD wird maßgeblich von der Häufigkeit einer Exazerbation bestimmt. Eine Exazerbation ist eine akute Verschlechterung der Beschwerden, die über die üblichen Tagesschwankungen hinausgeht und längere Zeit anhält. Die Patienten berichten über zunehmende Kurzatmigkeit, und dass sie bereits mehrfach am Tag ihre Bedarfstherapie benötigt haben.

\section{Merke}

Bei einer Exazerbation verschlechtern sich die Beschwerden akut - mehr als bei den typischen Tagesschwankungen üblich.

\section{Auslöser}

Auslöser für eine Exazerbation sind häufig akute Infekte. Hinter einer scheinbaren exazerbierten COPD können aber auch andere internistische Erkrankungen stecken (s. Differenzialdiagnosen).

\section{Anamnese}

Dazu hat sich das SAMPLER-Schema bewährt (Symptome, Allergien, Medikamente, Präerkrankungen, Letzte Mahlzeit, Ereignis und Risikofaktoren). Im nächsten Schritt erfolgt eine kurze Untersuchung des Patienten (ABCDEKonzept).

\section{Körperliche Untersuchung}

Bei der körperlichen Untersuchung erheben Sie zunächst die Atemfrequenz (normal 12 - 16 Atemzüge/min).

- Von einer Tachypnoe spricht man ab einer AF > 20/min.

- Unter Umständen sind eine Zyanose, juguläre oder interkostale Einziehungen bei der Inspiration erkennbar sowie der Einsatz der Atemhilfsmuskulatur.

- Bei der Auskultation fällt häufig ein Giemen und Brummen auf, evtl. auch feuchte Rasselgeräusche.

- Liegt ein Lungenemphysem mit schwerer Überblähung vor, hört man ein abgeschwächtes Atemgeräusch (s. u.). Perkutorisch ist der Klopfschall hypersonor.

- Tab. 5 zeigt, wie man die Schwere einer Exazerbation abschätzen kann.

\section{Differenzialdiagnosen}

Bei einer akuten Verschlechterung der COPD sollten Sie differenzialdiagnostisch auch an eine kardiale Insuffizienz (z. B. durch schwere Herzrhythmusstörungen), einen entgleisten Hypertonus und ein akutes Koronarsyndrom (ACS) denken. Lungenembolie, Fremdkörperaspiration sowie Pneumothorax sind auszuschließen. Gerade bei einem hohen Einsatz an Bronchodilatatoren ist eine Hypokaliämie möglich, die ebenso schwere Herzrhythmusstörungen zur Folge haben kann. Gelegentlich entwickeln COPD-Patienten eine chronische Rechtsherzinsuffizienz, die dekompensieren kann. 
- Tab. 5 Schweregrad der akuten Kurzatmigkeit bei COPD.

\begin{tabular}{|l|l|l|l|}
\hline & Mäßig & Schwer & Lebensbedrohlich \\
\hline Giemen und Brummen & deutlich & deutlich & sehr leise \\
\hline Sprache & wenig eingeschränkt & nur unvollständige Sätze & unfähig zu sprechen \\
\hline $\mathrm{pCO}_{2}$ & $\begin{array}{l}\text { Patient antwortet adäquat auf } \\
\text { Fragen }\end{array}$ & Patient wirkt verlangsamt & $\begin{array}{c}\text { Patient ist verwirrt/nicht } \\
\text { ansprechbar }\end{array}$ \\
\hline $\mathrm{SpO}_{2}{ }^{*}$ & $>90 \%$ & $>90 \%$ & $<90 \%$ \\
\hline $\mathrm{Herzfrequenz}$ & $\uparrow \uparrow$ & $\uparrow \uparrow$ & $\uparrow \uparrow$ oder $\downarrow$ \\
\hline $\mathrm{Blutdruck}$ & $\leftrightarrow \uparrow$ & $\leftrightarrow$ & $\downarrow$ \\
\hline $\mathrm{Atemfrequenz} \mathrm{pro} \mathrm{min}$ & $<25$ & $>25$ & $>25$ \\
\hline $\mathrm{SpO}_{2}$ : Sauerstoffkonzentration mit Pulsoximeter gemessen & & \\
\hline
\end{tabular}

\section{Merke}

Grundsätzlich sollten Sie bei akut auftretender Dyspnoe auch an eine Anaphylaxie, ein Inhalationstrauma sowie ein Hyperventilationssyndrom denken.

\section{Therapie der Exazerbation}

\section{Erste Maßnahmen}

Die Akuttherapie nach Eintreffen des Rettungsdienstes richtet sich nach den Beschwerden und nach den erhobenen Befunden. An erster Stelle steht die Sauerstoffgabe. Ziel ist eine Sauerstoffsättigung von $>90 \%$. Eine atemerleichternde Lagerung wird vom Patienten bevorzugt, oft wird die Atemhilfsmuskulatur genutzt. Eine Möglichkeit ist der Kutschersitz.

\section{PRAXIS/MAßNAHMEN \\ Tipp für die Praxis}

Manchmal besteht bereits eine Überlastung der Atemmuskelpumpe. Dies führt zu einer noch nicht erkannten Erhöhung des Blut- $\mathrm{CO}_{2}$ (Hyperkapnie). Daher sollten Sie die Bewusstseinslage des Patienten regelmäßig überprüfen. Die eher geringe Wahrscheinlichkeit einer $\mathrm{CO}_{2}$-Narkose sollte aber nicht zu einer ineffektiven Sauerstoffgabe führen. Häufig ist eine Sauerstoffmaske nötig, da viele Patienten bei Kurzatmigkeit zu einer Mundatmung neigen. Einen kurzfristigen Anstieg des $\mathrm{CO}_{2}$ im Blut kann man dabei tolerieren.

Die COPD ist oft mit einem instabilen Bronchialsystem verbunden. Bei starker Ausatmung kollabieren die instabilen Bronchien. Die dahinter befindliche Luft lässt sich nicht vollständig ausatmen. Beim nächsten Atemzug öffnen sich die Bronchien wieder und Luft strömt in die noch nicht entlüfteten Räume ein. Der Rückstau der noch nicht ausgeatmeten Luft führt zu einer dynamischen Überblähung mit zunehmender Reduktion des Atemvolumens. Die Kurzatmigkeit verstärkt sich. Ein deutlich abgeschwächtes Atemgeräusch, auch als „silent chest“ bezeichnet, ist Ausdruck dieser Überblähung.

\section{PRAXIS/MAßNAHMEN}

Tipp für die Praxis

Um einen Kollaps der Bronchien zu verhindern, sollten Sie den Patienten motivieren, die Lippenbremse einzusetzen. Dabei soll er durch leicht geschlossene Lippen ausatmen. Infolge des dadurch erzeugten positiven Drucks in der Lunge stabilisieren sich die Bronchien (innere Schienung).

\section{Inhalation}

Die schwere Dyspnoe verhindert häufig, dass der Patient die vorhandenen inhalativen Medikamente effektiv einsetzen kann. Hilfreich ist dann die Verwendung eines Spacers ( $\triangleright$ Abb. 2). Unter anderem können auch Bronchodilatatoren (Salbutamol, Atrovent) über einen Maskenvernebler ( $\boldsymbol{A} \mathbf{A b b}$. $\mathbf{3}$ ) zum Einsatz kommen, der an den Sauerstoff angeschlossen wird. Der Flow sollte dabei mindestens $6 \mathrm{l} / \mathrm{min}$ betragen, bei einem Flow $>8 \mathrm{l} / \mathrm{min}$ könnte sich allerdings der Schlauch aufgrund des hohen Drucks vom Vernebler lösen.

\section{Kortison}

Die systemische Gabe von Glukokortikoiden (Kortison) wird bei der Exazerbation der COPD als Initialtherapie empfohlen. Je frühzeitiger die systemische Gabe von Kortisonpräparaten erfolgt, desto größer ist der Effekt. Eine erste Besserung der Kurzatmigkeit können Sie frühestens 30 - 60 min nach i. v. Gabe erwarten. Im Allgemeinen ist die Gabe von 50 oder 100 mg Methylprednisolon i. v. ausreichend. 
- Tab. 6 Medikamentöse Therapie der COPD.

\begin{tabular}{|c|c|}
\hline Medikament & Anwendung \\
\hline inhalative Sympathomimetika & $\begin{array}{l}\text { initial } 4 \text { Hübe, dann } 2 \text { Hübe alle } \\
10 \text { min unter Pulskontrolle }\end{array}$ \\
\hline $\begin{array}{l}\text { Anticholinergika } \\
\text { (Ipratropiumbromid) }\end{array}$ & $\begin{array}{l}\text { initial } 4 \text { - } 8 \text { Hübe, dann } 2 \text { Hübe } \\
\text { alle } 20 \text { min }\end{array}$ \\
\hline \multirow[t]{2}{*}{ Prednisolon } & $40 \mathrm{mg}$ Tbl. oral \\
\hline & $100 \mathrm{mg}$ i. v. \\
\hline $\begin{array}{l}\text { Salbutamol Amp./Ipratropium- } \\
\text { bromid Amp. }\end{array}$ & $\begin{array}{l}\text { jeweils } 1 \text { Amp. über Masken- } \\
\text { vernebler }\end{array}$ \\
\hline $\begin{array}{l}\left.\text { Terbutalin (Bricanyl }{ }^{\circledR}\right) \text { Amp. }(1 \mathrm{ml}) \\
0,5 \mathrm{mg}\end{array}$ & $\begin{array}{l}\text { 1/2-1 Amp. s. c., wiederholen } \\
\text { nach } 15-20 \text { min möglich }\end{array}$ \\
\hline
\end{tabular}

Viele Patienten werden im Rahmen von COPD-Schulungen von ihren behandelnden Lungenfachärzten mit Prednisolon-Tabletten für den Notfall ausgestattet. Auch davon kann man $40 \mathrm{mg}$ als Akuttherapie verabreichen.

\section{Salbutamol}

Salbutamol wirkt direkt an den $\beta_{2}$-Rezeptoren der Bronchialmuskulatur: Es entspannt die glatte Muskulatur und führt somit zur Erweiterung der Bronchien. Es kommt sowohl bei der Therapie einer COPD als auch beim Asthma bronchiale zum Einsatz. Als Nebenwirkung können eine erhöhte Herzfrequenz und eine Hypokaliämie auftreten.

\section{Atrovent}

Atrovent (Ipratropiumbromid) ist ein kurz wirksamer Muskarinrezeptor-Antagonist (SAMA). Es ist ein Derivat des Atropins und blockiert die Acetylcholinrezeptoren, deren Stimulation ansonsten die Bronchialmuskulatur verengt. Somit kommt es bei inhalativer Anwendung zu einer Bronchodilatation. Atrovent wird bei der COPD und selten beim Asthma bronchiale eingesetzt. Die Dosierung ist $>$ Tab. 6 zu entnehmen.

\section{Terbutalin und Reproterol}

In der Notfallversorgung kommen gelegentlich auch Terbutalin (Bricanyl ${ }^{\circledR}$ ) und Reproterol (Bronchospasmin ${ }^{\circledR}$ ) zum Einsatz. Beide Substanzen sind Bronchodilatatoren aus der Gruppe der $\beta_{2}$-Sympathomimetika. Die Wirkung beruht auf der selektiven Bindung an $\beta_{2}$-Rezeptoren der Bronchialmuskulatur. Sie lassen sich v. a. alternativ einsetzen, wenn die inhalative Therapie von Salbutamol über ein Dosieraerosol nicht möglich ist oder eine Verneblermaske nicht toleriert wird.

Cave

Der Einsatz von $\beta_{2}$-Mimetika (Salbutamol, Terbutalin und Reproterol) weist bei der Behandlung der exazerbierten COPD gelegentlich nur einen geringen Effekt

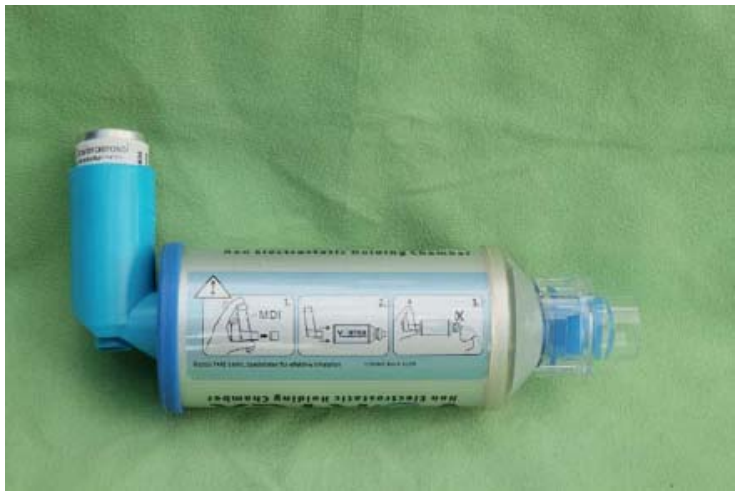

- Abb. 2 Ein Spacer kann das Verwenden eines Dosieraerosols erleichtern.

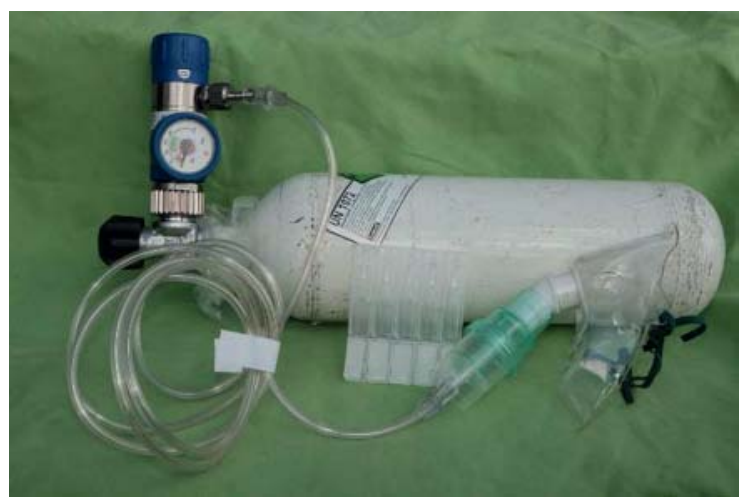

- Abb. 3 Bei Atemnot lässt sich auch ein an den Sauerstoff angeschlossener Maskenvernebler mit Bronchodilatatoren verwenden.

auf. Dies liegt z. T. daran, dass es durch den langen und intensiven Gebrauch der $\beta_{2}$-Mimetika in inhalativer Form (Salbutamol Dosieraerosol) bereits zu einer Downregulation der $\beta$-Rezeptoren gekommen ist. Außerdem sind die im Rahmen der akuten Exazerbation durch die schon eingesetzten Medikamente die $\beta$-Rezeptoren bereits belegt. An Nebenwirkungen sind immer Unruhe, Tremor, Palpitationen und Anstieg der Herzfrequenz zu bedenken, bei Terbutalin u. U. auch eine Absenkung des Blutdrucks.

\section{Magnesium}

Ein weiteres Reservemedikament in der präklinischen Therapie ist Magnesium. Es entfaltet seinen therapeutischen Effekt über eine Relaxation der glatten Muskulatur. Die i. v. Gabe sollte obligatorisch unter EKG-Monitoring erfolgen.

\section{Theophyllin}

Theophyllin wird bei akuter Exazerbation von den nationalen und internationalen Leitlinien für COPD-Patienten aufgrund der geringen therapeutischen Breite nur eingeschränkt empfohlen. In Kombination mit $\beta_{2}$-Sympatho- 
mimetika hat Theophyllin nur einen minimalen zusätzlichen bronchodilatatorischen Effekt. Kardiale Nebenwirkungen und schwere Herzrhythmusstörungen können auftreten.

\section{Nichtinvasive Beatmung}

Falls sich trotz der medikamentösen Therapie die Situation des Patienten nicht verbessert, können Sie eine nichtinvasive Beatmung (NIV) erwägen. Dadurch lässt sich eine deutliche Verbesserung beim Gasaustausch erreichen. Einerseits wird durch die NIV die sich erschöpfende Atemmuskulatur unterstützt, andererseits der Kollaps der Bronchien bei der Ausatmung verhindert. In Studien konnte gezeigt werden, dass sich durch den Einsatz der nichtinvasiven Beatmung die Sterblichkeit und die Verweildauer im Krankenhaus senken ließen.

\section{Indikation}

Die Indikation zur NIV ergibt sich bei zunehmender respiratorischer Erschöpfung (Zunahme der Atemfrequenz), beginnender kardiovaskulärer Dekompensation und bei abnehmender Vigilanz.

Die NIV ist generell kein Ersatz für eine invasive Beatmung. Sie hat sich jedoch gerade beim frühzeitigen, präklinischen Einsatz bei der akuten respiratorischen Insuffizienz durch ein kardiogenes Lungenödem sowie bei der exazerbierten COPD bewährt.

\section{Kontraindikationen}

Absolute Kontraindikationen sind:

- fehlende Spontanatmung oder Schnappatmung

- funktionelle oder fixierte Verlegung der Atemwege

- gastrointestinale Blutungen

Relative Kontraindikationen sind:

- Koma

- ausgeprägte Agitation

- massiver Sekretverhalt

- hämodynamische Instabilität

- Inakzeptanz durch den Patienten

\section{Monitoring}

An Gerätschaften ist neben einem Respirator eine Nasenoder Mund-Nasen-Maske erforderlich. Am häufigsten kommt die Mund-Nasen-Maske zum Einsatz.
Das Monitoring während des gesamten Transports erfolgt mittels Überwachung von

- $\mathrm{SpO}_{2}$,

- Atemfrequenz,

- Blutdruck,

- EKG und

- Beobachtung des Patienten (Vigilanz, Erschöpfung der Atemmuskulatur).

Ein erfolgreicher Einsatz der NIV zeigt sich in einer Abnahme der Dyspnoe, Atem- und Herzfrequenz sowie einer Besserung der Vigilanz und der Oxygenierung. Bei anhaltender muskulärer Erschöpfung, Anstieg der Atemfrequenz $>40 / \mathrm{min}$, anhaltender Hypoxämie und Kreislaufinstabilität sollte man die invasive Beatmung (ITN) in Erwägung ziehen.

\section{Merke}

Die Therapie der akuten Exazerbation besteht in Sauerstoffgabe, wiederholter Gabe von inhalativen Bronchodilatatoren, systemischer Gabe von Kortison und $\beta-\mathrm{Mi}$ metika sowie dem Einsatz der NIV.

\section{KERNAUSSAGEN}

- Die akute Exazerbation einer COPD ist ein erhebliches gesundheitliches Risiko.

- Da häufig ältere Patienten davon betroffen sind und bei COPD-Patienten häufig mehrere relevante Begleiterkrankungen vorliegen, ist die Exazerbation mit einer hohen Mortalität behaftet.

- Die Sterblichkeit steigt mit einer Intensivpflichtigkeit und bei wiederholten Krankenhausaufenthalten an.

- Aufgabe des Rettungsdienstes ist das rasche Sichern der Oxygenierung, u. U. durch den frühzeitigen Einsatz der nichtinvasiven Beatmung sowie die medikamentöse Therapie durch den Notarzt.

- Während des gesamten Einsatzes ist eine engmaschige Kontrolle der Vitalparameter und der Vigilanz des Patienten nötig.

\section{Interessenkonflikt}

Medizinische Vorträge im Rahmen von Veranstaltungen der Firmen Chiesi, Boehringer Ingelheim, Berlin-Chemie, Novartis und AstraZeneca, Beratertätigkeiten bei Chiesi, Boehringer Ingelheim, AstraZeneca und Berlin-Chemie. 


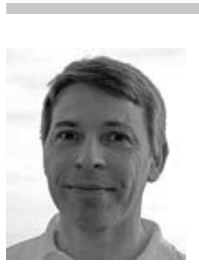

\section{Jochen Hinrichs-Pavlik}

Ist Facharzt für Innere Medizin/Pneumologie und Notfallmedizin in eigener Praxis in Heidelberg, Leiter des Arbeitskreises nordbadischer Pneumologen. Ehrenamtlich ist er in der DRKBereitschaft Neckargemünd-Wiesenbach tätig und Zugarzt der SEG 3, Rhein-Neckar.

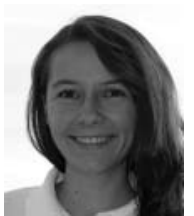

\section{Jule Pavlik}

Studiert in Marburg Medizin und ist als Rettungssanitäterin in der DRK-Bereitschaft Neckargemünd-Wiesenbach tätig.

\section{Korrespondenzadresse}

\section{Literatur}

[1] Beutel B, Greulich T, Vogelmeier C et al. Übersicht COPD. Der Pneumologe 2017; 1: 35-43

[2] Groeben H, Kullmann HJ. Akuter Asthmaanfall und exazerbierte chronisch obstruktive Lungenerkrankung. Notfall Rettungsmed 2011; 14: $61-76$

[3] Global Initiative for Chronic Obstructive Lung Disease. http:// goldcopd.org/gold-2017-global-strategy-diagnosis-management-prevention-copd (Stand: 03.08.2017)

\section{Bibliografie}

DOI https://doi.org/10.1055/s-0043-110109 retten 2017; 6: 298-308

(c) Georg Thieme Verlag KG, Stuttgart · New York ISSN 2193-2387

Jochen Hinrichs-Pavlik

FA für Inn. Med./Pneumologie/Notfallmedizin

Bergheimerstr. 3

69115 Heidelberg

praxis.hinrichs-pavlik@web.de 


\section{Punkte samneln auf CEEthienede}

口店口

Diese Fortbildungseinheit ist 12 Monate online für die Teilnahme verfügbar.

Sollten Sie Fragen zur Online-Teilnahme haben, finden Sie unter cme.thieme.de/hilfe

eine ausführliche Anleitung. Wir wünschen viel Erfolg beim Beantworten

der Fragen!

Unter https://eref.thieme.de/ZZX8TGD oder über den QR-Code kommen Sie direkt zum Artikel zur Eingabe der Antworten.

\section{Frage 1}

Welche Aussage ist korrekt? Die chronisch-obstruktive Lungenerkrankung (COPD) ...

A ist dem Asthma gleichzusetzen.

B beruht auf einer stark variablen Obstruktion.

$\mathrm{C}$ ist durch eine chronisch fortschreitende Verengung der Bronchien gekennzeichnet.

D ist durch inhalative Medikamente vollständig reversibel.

E betrifft ausschließlich junge Patienten.

\section{Frage 2}

Welche Aussage trifft zu?

A Die COPD ist eine seltene Erkrankung ( $<1 \%)$.

B Eine COPD wird häufig durch langjährigen Nikotinkonsum ausgelöst.

C Ursache für eine COPD ist meist eine Allergie.

D Die COPD geht nur im Rahmen einer Exazerbation mit Kurzatmigkeit einher.

E Typisch für eine COPD ist eine chronische Obstruktion der Blutgefäße.

\section{Frage 3}

Wann ist die Indikation zur nichtinvasiven Beatmung/Ventilation (NIV) zu stellen?
A bei fehlender Spontanatmung
B bei massivem Sekretverhalt in den Bronchien
$C$ bei gastrointestinalen Blutungen
D bei zunehmender respiratorischer Erschöpfung
$\mathrm{E}$ bei einer Verlegung der Atemwege

\section{Frage 4}

Welche Medikamente werden zur Therapie der COPD eingesetzt?
A Bronchodilatatoren (Bronchospasmolytika)
B Nitrospray
C Antiallergika (Antihistaminika)
D $\beta$-Blocker
E Diuretika

\section{Frage 5}

Welche Aussage stimmt?

A Kortison wird schon bei leichter COPD eingesetzt.

B Theophyllin ist bei jedem Schweregrad einer COPD indiziert.

C Bei einer CO2-Narkose fällt das $\mathrm{CO} 2 \mathrm{ab}$.

D $\beta 2$-Sympatomimetika führen häufig zu einer Mundtrockenheit.

E Bronchodilatatoren können als Nebenwirkung die Pulsfrequenz und den Blutdruck erhöhen.

\section{Frage 6}

Welche Differenzialdiagnose ist bei einer vermuteten COPDExazerbation weniger wahrscheinlich?
A kardiale Insuffizienz
B entgleister arterieller Hypertonus
C Lungenembolie
D Nierenkolik
E akutes Koronarsyndrom

\section{Frage 7}

Welche Maßnahmen kommen bei der Versorgung einer akuten COPD-Exazerbation nicht zum Einsatz?
A Sauerstoffgabe
B wiederholte Gabe von inhalativen Bronchodilatatoren
C konstante Überwachung von Vitalzeichen und Bewusstseinslage
D flache Lagerung
E Anleitung zur Lippenbremse

\section{Frage 8}

Welche Komplikation kann bei einer COPD-Exazerbation auftreten?

A Anstieg der Herzfrequenz durch hohe Dosierungen von inhalativen Bronchodilatatoren

B Bradykardie nach Einsatz von Theophyllin

C Abfall von $\mathrm{CO}_{2}$ unter Gabe von Sauerstoff

D immer ein Abfall des Blutzuckers

E ein lleus

- Weitere Fragen auf der folgenden Seite... 


\section{Punkte sammeln auf CEE.thieme.de}

\section{Frage 9}

Was müssen Sie bei Patienten mit einer exazerbierten COPD beachten?
A Meist sind weibliche Patienten betroffen.
B Eine Exazerbation stellt keine Notarzt-Indikation dar.
C Die Exazerbation lässt sich immer ambulant beherrschen.
D Die häufig bestehenden Begleiterkrankungen können den akuten Notfall negativ beeinflussen.
E Eine COPD ist harmlos und es ist nichts weiter zu beachten.

\section{Frage 10}

Welche Aussage trifft nicht zu?

A Durch eine Überlastung der Atemmuskelpumpe kann es zu einem Anstieg von $\mathrm{CO} 2$ kommen.

B Unter Umständen ist bei der Exazerbation die Einleitung einer nichtinvasiven Beatmung (NIV) nötig.

C Die Exazerbation geht mit einer geringen Mortalität einher.

D Bronchodilatatoren lassen sich auch über einen Maskenvernebler einsetzen.

E Durch eine dynamische Überblähung reduziert sich das Atemvolumen. 